\title{
ON MANY-SORTED ALGEBRAIC CLOSURE OPERATORS
}

\author{
J. CLIMENT VIDAL AND J. SOLIVERES TUR
}

\begin{abstract}
A theorem of Birkhoff-Frink asserts that every algebraic closure operator on an ordinary set arises, from some algebraic structure on the set, as the operator that constructs the subalgebra generated by a subset. However, for many-sorted sets, i.e., indexed families of sets, such a theorem is not longer true without qualification. We characterize the corresponding many-sorted closure operators as precisely the uniform algebraic operators.
\end{abstract}

Some theorems of ordinary universal algebra can not be automatically generalized to many-sorted universal algebra, e.g., Matthiessen [5] proves that there exist many-sorted algebraic closure systems that can not be concretely represented as the set of subalgebras of a many-sorted algebra. As is well known, according to a representation theorem of Birkhoff and Frink [1], this is not so for the single-sorted algebraic closure systems.

In [2] it was obtained a concrete representation for the so-called many-sorted uniform 2-algebraic closure operators. However, as will be proved below, confirming a conjecture by A. Blass in his review of [2], the main result in [2] remains true if we delete from the above class of many-sorted operators the condition of 2-algebraicity. Therefore a many-sorted algebraic closure operator will be concretely representable as the set of subalgebras of a many-sorted algebra iff it is uniform. We point out that the proof we offer follows substantially that in Grätzer [4] for the single-sorted case, but differs from it, among others things, by the use we have to make, on the one hand, of the concept of uniformity, missing in the single-sorted case, and, on the other hand, of the Axiom of Choice, because of the lack, in the many-sorted case, of a canonical choice in the definition of the many-sorted operations.

In what follows we use, for a set of sorts $S$ and an $S$-sorted signature $\Sigma$, the concept of many-sorted $\Sigma$-algebra and subalgebra in the standard meaning, see e.g., [3].

To begin with, as for ordinary algebras, also the set of subalgebras of a manysorted algebra is an algebraic closure system.

Proposition 1. Let $\underline{A}$ be a many-sorted $\Sigma$-algebra. Then the set of all subalgebras of $\underline{A}$, denoted by $\operatorname{Sub}(\underline{A})$, is an algebraic closure system on $A$, i.e., we have

(1) $A \in \operatorname{Sub}(\underline{A})$.

(2) If $I$ is not empty and $\left(X^{i}\right)_{i \in I}$ is a family in $\operatorname{Sub}(\underline{A})$, then $\bigcap_{i \in I} X^{i}$ is also in $\operatorname{Sub}(A)$.

(3) If I is not empty and $\left(X^{i}\right)_{i \in I}$ is an upwards directed family in $\operatorname{Sub}(\underline{A})$, then $\bigcup_{i \in I} X^{i}$ is also in $\operatorname{Sub}(\underline{A})$.

However, as we will prove later on, in the many-sorted case the many-sorted algebraic closure operator canonically associated to the algebraic closure system of the subalgebras of a many-sorted algebra has an additional and characteristic property, that of being uniform.

Date: November 22, 2004.

1991 Mathematics Subject Classification. Primary: 08A30, 06A15; Secondary: 08A68.

Key words and phrases. Many-sorted algebra, support, many-sorted algebraic closure operator, many-sorted uniform algebraic closure operator. 
Now we recall the concept of support of a sorted set and that of many-sorted algebraic closure operator on a sorted set, essentials to define that of many-sorted algebraic uniform closure operator.

Definition 1. Let $A$ be an $S$-sorted set. Then the support of $A$, denoted by $\operatorname{supp}(A)$, is the subset $\left\{s \in S \mid A_{s} \neq \varnothing\right\}$ of $S$.

Definition 2. Let $A$ be an $S$-sorted set. A many-sorted algebraic closure operator on $A$ is an operator $J$ on $\operatorname{Sub}(A)$, the set of all $S$-sorted subsets of $A$, such that, for every $X, Y \subseteq A$, satisfies:

(1) $X \subseteq J(X)$, i.e., $J$ is extensive.

(2) If $\bar{X} \subseteq Y$, then $J(X) \subseteq J(Y)$, i.e., $J$ is isotone.

(3) $J(J(X))=J(X)$, i.e., $J$ is idempotent.

(4) $J(X)=\bigcup_{F \in \operatorname{Sub}_{\mathrm{f}}(X)} J(F)$, i.e., $J$ is algebraic, where a part $F$ of $X$ is in $\operatorname{Sub}_{\mathrm{f}}(X)$, the set of finite $S$-sorted subsets of $X$, iff $\operatorname{supp}(F)$ is finite and, for every $s \in \operatorname{supp}(F), F_{s}$ is finite.

A many-sorted algebraic closure operator $J$ on $A$ is uniform iff, for $X, Y \subseteq A$, from $\operatorname{supp}(X)=\operatorname{supp}(Y)$, follows that $\operatorname{supp}(J(X))=\operatorname{supp}(J(Y))$.

Definition 3. Let $\underline{A}$ be a many-sorted $\Sigma$-algebra. We denote by $\operatorname{Sg}_{\underline{A}}$ the manysorted algebraic closure operator on $A$ canonically associated to the algebraic closure system $\operatorname{Sub}(\underline{A})$. If $X \subseteq A, \operatorname{Sg}_{A}(X)$ is the subalgebra of $\underline{A}$ generated by $X$.

Next, as for ordinary algebras, we define for a many-sorted $\Sigma$-algebra $\underline{A}$ an operator on $\operatorname{Sub}(A)$ that will allow us to obtain, for every subset of $A$, by recursion, an $\mathbb{N}$-ascending chain of subsets of $A$ from which, taking the union, we will obtain an equivalent, but more constructive, description of the subalgebra of $\underline{A}$ generated by a subset of $A$. Moreover, we will make use of this alternative description to prove the uniformity of the operator $\mathrm{Sg}_{A}$ and also in the proof of the representation theorem.

Definition 4. Let $\underline{A}=(A, F)$ be a many-sorted $\Sigma$-algebra.

(1) We denote by $\mathrm{E}_{\underline{A}}$ the operator on $\operatorname{Sub}(A)$ that assigns to an $S$-sorted subset $X$ of $A, \mathrm{E}_{\underline{A}}(X)=X \cup\left(\bigcup_{\sigma \in \Sigma, s} F_{\sigma}\left[X_{\operatorname{ar}(\sigma)}\right]\right)_{s \in S}$, where, for $s \in S, \Sigma_{., s}$ is the set of all many-sorted formal operations $\sigma$ such that the coarity of $\sigma$ is $s$ and for $\operatorname{ar}(\sigma)=\left(s_{j}\right)_{j \in m} \in S^{\star}$, the arity of $\sigma, X_{\operatorname{ar}(\sigma)}=\prod_{j \in m} X_{s_{j}}$.

(2) If $X \subseteq A$, then the family $\left(\mathrm{E}_{\underline{A}}^{n}(X)\right)_{n \in \mathbb{N}}$ in $\operatorname{Sub}(A)$ is such that $\mathrm{E}_{\underline{A}}^{0}(X)=X$ and $\mathrm{E}_{A}^{n+1}(X)=\mathrm{E}_{\underline{A}}\left(\mathrm{E}_{A}^{n}(X)\right)$, for $n \geq 0$.

(3) We denote by $\mathrm{E}_{A}^{\omega}$ the operator on $\operatorname{Sub}(A)$ that assigns to an $S$-sorted subset $X$ of $A, \mathrm{E}_{\underline{A}}^{\omega}(X)=\bigcup_{n \in \mathbb{N}} \mathrm{E}_{A}^{n}(X)$

Proposition 2. Let $\underline{A}$ be a many-sorted $\Sigma$-algebra and $X \subseteq A$. Then we have that $\operatorname{Sg}_{\underline{A}}(X)=\mathrm{E}_{\underline{A}}^{\omega}(X)$.

Proof. See [2]

Proposition 3. Let $\underline{A}$ be a many-sorted $\Sigma$-algebra and $X, Y \subseteq A$. Then we have that

(1) If $\operatorname{supp}(X)=\operatorname{supp}(Y)$, then, for every $n \in \mathbb{N}, \operatorname{supp}\left(\mathrm{E}_{\underline{A}}^{n}(X)\right)=\operatorname{supp}\left(\mathrm{E}_{\underline{A}}^{n}(Y)\right)$.

(2) $\operatorname{supp}\left(\operatorname{Sg}_{\underline{A}}(X)\right)=\bigcup_{n \in \mathbb{N}} \operatorname{supp}\left(\mathrm{E}_{\underline{A}}^{n}(X)\right)$.

(3) If $\operatorname{supp}(X)=\operatorname{supp}(Y)$, then $\operatorname{supp}\left(\operatorname{Sg}_{\underline{A}}(X)\right)=\operatorname{supp}\left(\operatorname{Sg}_{\underline{A}}(Y)\right)$.

Therefore the many-sorted algebraic closure operator $\mathrm{Sg}_{\underline{A}}$ is uniform.

Proof. See [2]

Finally we prove the representation theorem for the many-sorted uniform algebraic closure operators, i.e., we prove that for an $S$-sorted set $A$ a many-sorted 
algebraic closure operator $J$ on $\operatorname{Sub}(A)$ has the form $\operatorname{Sg}_{A}$, for some $S$-sorted signature $\Sigma$ and some many-sorted $\Sigma$-algebra $\underline{A}$ if $J$ is uniform.

Theorem 1. Let $J$ be a many-sorted algebraic closure operator on an $S$-sorted set $A$. If $J$ is uniform, then $J=\operatorname{Sg}_{\underline{A}}$ for some $S$-sorted signature $\Sigma$ and some many-sorted $\Sigma$-algebra $\underline{A}$.

Proof. Let $\Sigma=\left(\Sigma_{w, s}\right)_{(w, s) \in S^{\star} \times S}$ be the $S$-sorted signature defined, for every $(w, s) \in S^{\star} \times S$, as follows:

$$
\Sigma_{w, s}=\left\{(X, b) \in \bigcup_{X \in \operatorname{Sub}(A)}\left(\{X\} \times J(X)_{s}\right) \mid \forall t \in S\left(\operatorname{card}\left(X_{t}\right)=|w|_{t}\right)\right\},
$$

where for a sort $s \in S$ and a word $w:|w| \longrightarrow S$ on $S$, with $|w|$ the lenght of $w$, the number of occurrences of $s$ in $w$, denoted by $|w|_{s}$, is $\operatorname{card}(\{i \in|w| \mid w(i)=s\})$.

We remark that for $(w, s) \in S^{\star} \times S$ and $(X, b) \in \bigcup_{X \in \operatorname{Sub}(A)}\left(\{X\} \times J(X)_{s}\right)$ the following conditions are equivalent:

(1) $(X, b) \in \Sigma_{w, s}$, i.e., for every $t \in S, \operatorname{card}\left(X_{t}\right)=|w|_{t}$.

(2) $\operatorname{supp}(X)=\operatorname{Im}(w)$ and, for every $t \in \operatorname{supp}(X), \operatorname{card}\left(X_{t}\right)=|w|_{t}$.

On the other hand, for the index set $\Lambda=\bigcup_{Y \in \operatorname{Sub}(A)}(\{Y\} \times \operatorname{supp}(Y))$ and the $\Lambda$-indexed family $\left(Y_{s}\right)_{(Y, s) \in \Lambda}$ whose $(Y, s)$-th coordinate is $Y_{s}$, precisely the $s$-th coordinate of the $S$-sorted set $Y$ of the index $(Y, s) \in \Lambda$, let $f$ be a choice function for $\left(Y_{s}\right)_{(Y, s) \in \Lambda}$, i.e., an element of $\prod_{(Y, s) \in \Lambda} Y_{s}$. Moreover, for every $w \in S^{\star}$ and $a \in \prod_{i \in|w|} A_{w(i)}$, let $M^{w, a}=\left(M_{s}^{w, a}\right)_{s \in S}$ be the finite $S$-sorted subset of $A$ defined as $M_{s}^{w, a}=\left\{a_{i} \mid i \in w^{-1}[s]\right\}$, for every $s \in S$.

Now, for $(w, s) \in S^{\star} \times S$ and $(X, b) \in \Sigma_{w, s}$, let $F_{X, b}$ be the many-sorted operation from $\prod_{i \in|w|} A_{w(i)}$ into $A_{s}$ that to an $a \in \prod_{i \in|w|} A_{w(i)}$ assigns $b$, if $M^{w, a}=X$ and $f\left(J\left(M^{w, a}\right), s\right)$, otherwise.

We will prove that the many-sorted $\Sigma$-algebra $\underline{A}=(A, F)$ is such that $J=$ $\mathrm{Sg}_{A}$. But before that it is necessary to verify that the definition of the manysorted operations is sound, i.e., that for every $(w, s) \in S^{\star} \times S,(X, b) \in \Sigma_{w, s}$ and $a \in \prod_{i \in|w|} A_{w(i)}, s \in \operatorname{supp}\left(J\left(M^{w, a}\right)\right)$ and for this it is enough to prove that $\operatorname{supp}\left(M^{w, a}\right)=\operatorname{supp}(X)$, because, by hypothesis, $J$ is uniform and, by definition, $b \in J(X)_{s}$.

If $t \in \operatorname{supp}\left(M^{w, a}\right)$, then $M_{t}^{w, a}$ is nonempty, i.e., there exists an $i \in|w|$ such that $w(i)=t$. Therefore, because $(X, b) \in \Sigma_{w, s}$, we have that $0<|w|_{t}=\operatorname{card}\left(X_{t}\right)$, hence $t \in \operatorname{supp}(X)$.

Reciprocally, if $t \in \operatorname{supp}(X),|w|_{t}>0$, and there is an $i \in|w|$ such that $w(i)=t$, hence $a_{i} \in A_{t}$, and from this we conclude that $M_{t}^{w, a} \neq \varnothing$, i.e., that $t \in \operatorname{supp}\left(M^{w, a}\right)$. Therefore, $\operatorname{supp}\left(M^{w, a}\right)=\operatorname{supp}(X)$ and, by the uniformity of $J, \operatorname{supp}\left(J\left(M^{w, a}\right)\right)=$ $\operatorname{supp}(J(X))$. But, by definition, $b \in J(X)_{s}$, so $s \in \operatorname{supp}\left(J\left(M^{w, a}\right)\right)$ and the definition is sound.

Now we prove that, for every $X \subseteq A, J(X) \subseteq \operatorname{Sg}_{A}(X)$. Let $X$ be an $S$-sorted subset of $A, s \in S$ and $b \in J(X)_{s}$. Then, because $\bar{J}$ is algebraic, $b \in J(Y)_{s}$, for some finite $S$-sorted subset $Y$ of $X$. From such an $Y$ we will define a word $w_{Y}$ in $S$ and an element $a_{Y}$ of $\prod_{i \in\left|w_{Y}\right|} A_{w_{Y}(i)}$ such that

(1). $Y=M^{w_{Y}, a_{Y}}$

(2). $(Y, b) \in \Sigma_{w_{Y}, s}$, i.e., $b \in J(Y)_{s}$ and, for all $t \in S$, $\operatorname{card}\left(Y_{t}\right)=\left|w_{Y}\right|_{t}$, and

(3). $a_{Y} \in \prod_{i \in\left|w_{Y}\right|} X_{w_{Y}(i)}$

then, because $F_{Y, b}\left(a_{Y}\right)=b$, we will be entitled to assert that $b \in \operatorname{Sg}_{\underline{A}}(X)_{s}$.

But taking into account that $Y$ is finite iff $\operatorname{supp}(Y)$ is finite and, for every $t \in \operatorname{supp}(Y), Y_{t}$ is finite, let $\left\{s_{\alpha} \mid \alpha \in m\right\}$ be an enumeration of $\operatorname{supp}(Y)$ and, for every $\alpha \in m$, let $\left\{y_{\alpha, i} \mid i \in p_{\alpha}\right\}$ be an enumeration of the nonempty $s_{\alpha}$-th coordinate, $Y_{s_{\alpha}}$, of $Y$. Then we define, on the one hand, the word $w_{Y}$ as the 
mapping from $\left|w_{Y}\right|=\sum_{\alpha \in m} p_{\alpha}$ into $S$ such that, for every $i \in\left|w_{Y}\right|$ and $\alpha \in m$, $w_{Y}(i)=s_{\alpha}$ iff $\sum_{\beta \in \alpha} p_{\beta} \leq i \leq \sum_{\beta \in \alpha+1} p_{\beta}-1$ and, on the other hand, the element $a_{Y}$ of $\prod_{i \in\left|w_{Y}\right|} A_{w_{Y}(i)}$ as the mapping from $\left|w_{Y}\right|$ into $\bigcup_{i \in\left|w_{Y}\right|} A_{w_{Y}(i)}$ such that, for every $i \in\left|w_{Y}\right|$ and $\alpha \in m, a_{Y}(i)=y_{\alpha, i-\sum_{\beta \in \alpha} p_{\beta}}$ iff $\sum_{\beta \in \alpha} p_{\beta} \leq i \leq \sum_{\beta \in \alpha+1} p_{\beta}-1$. From these definitions follow (1), (2) and (3) above. Let us observe that (1) is a particular case of the fact that the mapping $M$ from $\bigcup_{w \in S^{\star}}\left(\{w\} \times \prod_{i \in|w|} A_{w(i)}\right)$ into $\operatorname{Sub}_{\mathrm{f}}(A)$ that to a pair $(w, a)$ assigns $M^{w, a}$ is surjective.

From the above and the definition of $F_{Y, b}$ we can affirm that $F_{Y, b}\left(a_{Y}\right)=b$, hence $b \in \operatorname{Sg}_{\underline{A}}(X)_{s}$. Therefore $J(X) \subseteq \operatorname{Sg}_{\underline{A}}(X)$.

Finally, we prove that, for every $X \subseteq A, \operatorname{Sg}_{A}(X) \subseteq J(X)$. But for this, by the Proposition 2, it is enough to prove that, for every subset $X$ of $A$, we have that $\mathrm{E}_{\underline{A}}(X) \subseteq J(X)$. Let $s \in S$ be and $c \in \mathrm{E}_{\underline{A}}(X)_{s}$. If $c \in X_{s}$, then $c \in J(X)_{s}$, because $J$ is extensive. If $c \notin X_{s}$, then, by the definition of $\mathrm{E}_{A}(X)$, there exists a word $w \in S^{\star}$, a many-sorted formal operation $(Y, b) \in \Sigma_{w, s}$ and an $a \in \prod_{i \in|w|} X_{w(i)}$ such that $F_{Y, b}(a)=c$. If $M^{w, a}=Y$, then $c=b$, hence $c \in J(Y)_{s}$, therefore, because $M^{w, a} \subseteq X, c \in J(X)_{s}$. If $M^{w, a} \neq Y$, then $F_{Y, b}(a) \in J\left(M^{w, a}\right)_{s}$, but, because $M^{w, a} \subseteq X$ and $J$ is isotone, $J\left(M^{w, a}\right)$ is a subset of $J(X)$, hence $F_{Y, b}(a) \in J(X)_{s}$. Therefore $\mathrm{E}_{A}(X) \subseteq J(X)$.

From this last Theorem and the Proposition 3. we obtain

Corollary 1. Let $J$ be a many-sorted algebraic closure operator on an $S$-sorted set $A$. Then $J=\operatorname{Sg}_{A}$ for some many-sorted $\Sigma$-algebra $\underline{A}$ iff $J$ is uniform.

\section{REFERENCES}

[1] G. Birkhoff and O. Frink, Representation of lattices by sets, Trans. Amer. Math. Soc., 64 (1948), pp. 299-316.

[2] J. Climent and L. Fernandino, On the relation between heterogeneous uniform 2-algebraic closure operators and heterogeneous algebras, Collec. Math., 40 (1990), pp 93-101; MR 92d:08009 (A. Blass).

[3] J. Goguen and J. Meseguer, Completeness of many-sorted equational logic, Houston Journal of Mathematics, 11 (1985), pp. 307-334.

[4] G. Grätzer, Universal algebra. 2nd ed., Springer-Verlag, 1979.

[5] G. Matthiessen, Theorie der Heterogenen Algebren, Mathematik-Arbeits-Papiere, Nr. 3, Universität, Bremen, 1976.

Universidad de Valencia, Departamento de Lógica y Filosofía de la Ciencia, E-46010 VAlencia, Spain

E-mail address: Juan.B.Climent@uv.es 